\title{
Automatic Scene Comparison and Matching in Multimodal Cytopathological Microscopic Images
}

Ropers, Sven-Olaf and Bell, André A and Würflinger, Thomas and Böcking, Alfred and Meyer-Ebrecht, Dietrich

Institute of Imaging and Computer Vision

RWTH Aachen University, 52056 Aachen, Germany

tel: +49 2418027860 , fax: +49 2418022200

web: www.lfb.rwth-aachen.de

in: IEEE International Conferene on Image Processing. ICIP 2005. See also BIBT $\mathrm{E}$ entry below.

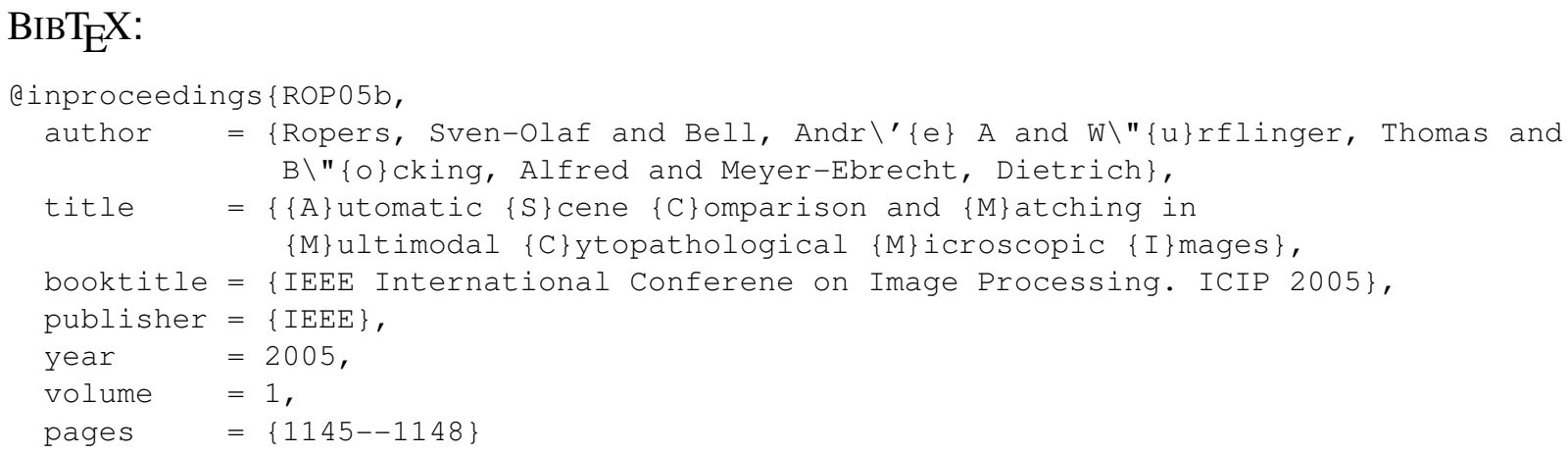

(C) 2005 IEEE. Personal use of this material is permitted. However, permission to reprint/republish this material for advertising or promotional purposes or for creating new collective works for resale or redistribution to servers or lists, or to reuse any copyrighted component of this work in other works must be obtained from the IEEE. 


\title{
AUTOMATIC SCENE COMPARISON AND MATCHING IN MULTIMODAL CYTOPATHOLOGICAL MICROSCOPIC IMAGES
}

\author{
S.-O. Ropers ${ }^{1}$, A. A. Bell ${ }^{1}$, T. Würflinger ${ }^{1}$, A. Böcking ${ }^{2}$ and D. Meyer-Ebrecht ${ }^{1}$ \\ Email: $\{$ sr,ab,tw,me $\} @ 1 f b . r w t h-a a c h e n . d e$ \\ ${ }^{1}$ Institute of Imaging and Computer Vision, RWTH Aachen University, Germany \\ ${ }^{2}$ Institute for Cytopathology, Heinrich-Heine-University, Düsseldorf, Germany
}

\begin{abstract}
The potential of cytopathologic diagnoses to detect cancer of a variety of types non-invasively, cost-efficiently and up to three years ahead of conventional histopathologic diagnoses can be increased by the application of adjuvant methods, i.e. the combination of different stainings. For further improvement of cytopathology we introduced Multimodal Cell Analysis (MMCA) which combines specific information about identical cells in different stainings successively applied to the same microscope slide. This requires a precise relocation and coregistration of individual cells under scrutiny. As a precondition for application in daily routine and screening settings the crucial relocation of cells has to be automated.

The paper describes a method for an automatic retrieval of images of cells which have already been selected and recorded in a preceding staining together with their coordinates. Due to inevitable mechanical inaccuracies the geometric match is insufficient. The comparison of nuclear constellations based on segmentations in both stains facilitates an automatic correction of the position even if there is a sub-scene matching only. The process furthermore generates the initial guess for the succeeding coregistration which thereby gains robustness. The success rate of the method described is about $85 \%$.
\end{abstract}

\section{INTRODUCTION}

Routine cancer diagnoses are nowadays based on histopathologic investigations of tissue specimens which are acquired in the course of an operation or biopsy. The tumour, however, must have reached a state in which it is already macroscopically visible. Non-invasively obtained single cells can be the clue to detect cancer in a much earlier state at lower cost [1]. A recent approach to automate and enhance the diagnostic process is the Multimodal Cell Analysis (MMCA),

\footnotetext{
The project is supported by the Viktor-and-Mirka-Pollak-Funds for Biomedical Engineering.
}

which utilises specimen (for example smears) on microscope slides for repetitive staining. The combination of specific information about identical cells in different stains yields multivariate statistics, which allow a further increase of diagnostic accuracy [2].

In order to relocate the cells after the specimen has been re-stained the images are stored in a database along with their positions. Due to mechanical tolerances in the gears of the microscope stage and because of inaccurate slide insertion, the multimodal image sets are not aligned properly. After the correction of the position with a global translation vector the displacement is still between 10 and 30 $\mu m$, corresponding to about 1-6 nuclei diameters. This displacement shall be corrected automatically. For the use of MMCA this is necessary to be done routinely. Hence it has to be robust as well as fast. In a first step scene knowledge is extracted from each image by a pre-segmentation. In a second step these scenes are compared and matched. If two scenes overlap only partially, the position is corrected respectively by moving the microscope stage, and the scenes are compared again.

\section{STATE OF THE ART}

The problem of multimodal scene matching is quite different from the well known problem of image registration. First of all, not necessarily all cell nuclei from the previously captured image lie directly in the current field of view (FOV). Hence sub-scenes have to be analysed and matched at runtime. The images have a rather homogeneous background, and a pixel based registration leads to local extrema and thus to false results. A content based approach can prevent this. The different appearance of identical scenes in different stains makes the registration of the images very difficult if the initial position is too far from the global maximum, even with the use of mutual information. A scene comparison can provide this initialisation for the final registration of the two images, which is necessary for a match with sub-pixel accuracy. A solution for these problems has not been found in literature. 


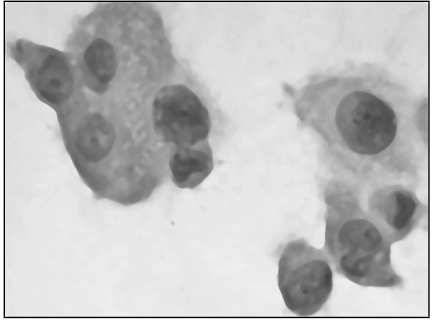

(a)

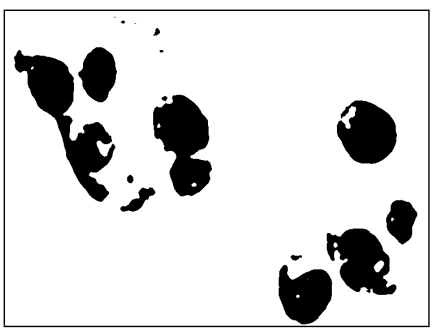

(d)

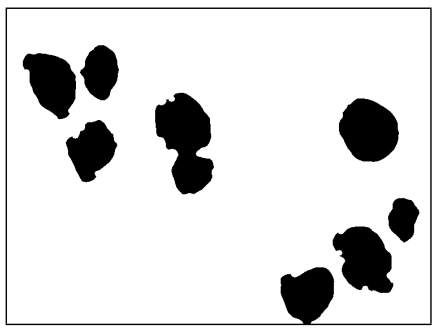

(g)

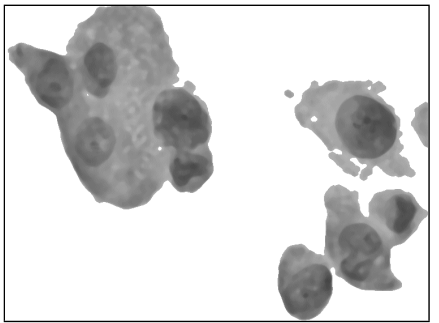

(b)

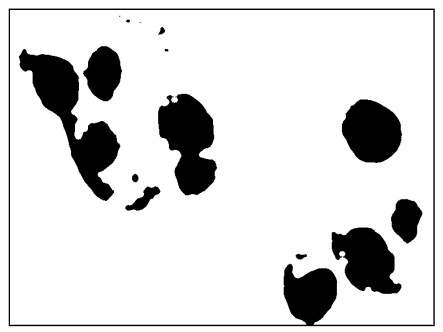

(e)

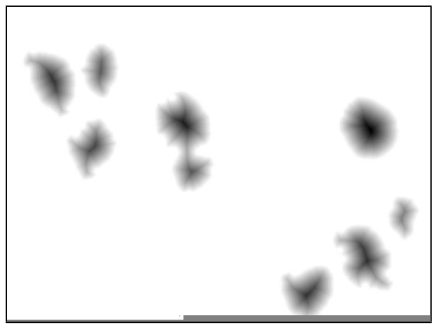

(h)

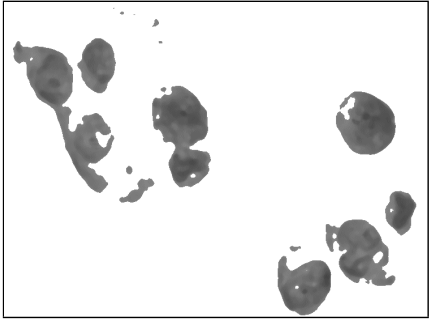

(c)

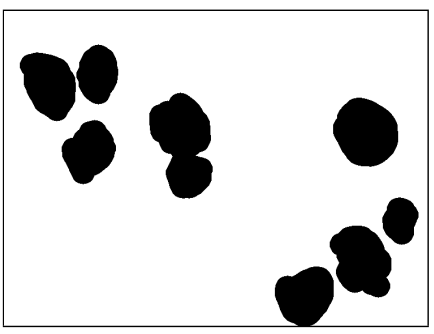

(f)

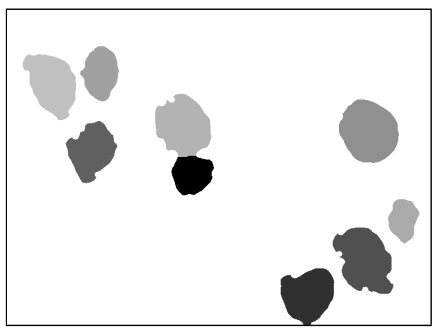

(i)

Fig. 1. Different steps of pre-segmentation: (a) Greyscaled source image, (b) Background masked out, (c) Plasma masked out, (d) Binary image, (e) After closing and flood filling, (f) Mask image, (g) AND-relation of (e) and (f), (h) Inverted distance transformed image, (i) Obtained scene

\section{EXPERIMENTAL SETUP AND MATERIAL}

The microscope workstation consists of a Leica DMLA light microscope, a 63x objective (Oil imm., NA 1.32), a 3-chip CCD RGB camera (JAI CV-M90, 768x576 px, 3x8 bit) and a PC for microscope control (xyz stage, etc.), image capture and analysis. The edge length of a pixel is $0.0992 \mu \mathrm{m}$ and thus the FOV is $76.2 \times 57.1 \mu \mathrm{m}^{2}$. The automatic image retrieval by means of scene comparison was applied to eight different samples (serous effusions) of both inflammatory and tumourous smears. For each sample about 200 reference images were available in a database in one or two of the following cytological stains: May-Grünwald-Giemsa (MGG), Feulgen (FEU) and Silver (SIL). Each reference image contained 12 nuclei on the average. Based on these existing images, overall 12 series with 2014 new images were retrieved in a subsequent stain. (6 series FEU referring to MGG, 3 series SIL ref. to FEU, 3 series SIL ref. to MGG).

\section{METHODS}

To retrieve the cell nuclei of the reference image in the current sample, the stage is repositioned according to the original coordinates. Then a global correction vector is applied to compensate for the overall displacement. After autofocus, a pre-segmentation is performed to detect the nuclei followed by a scene comparison for matching the scenes. If the result of the scene comparison is negative, the search is expanded to the surrounding area. Pre-segmentation and scene comparison are described in detail below.

\subsection{PRE-SEGMENTATION}

Since the segmentation takes place at runtime and because the succeeding scene comparison applies to the scene as a whole, a fast, not necessarily exact segmentation of the nuclei is used which will be called pre-segmentation.

The almost monochromatic images are transformed to greyscale images (see Fig. 1 (a)) using stain-specific, em- 


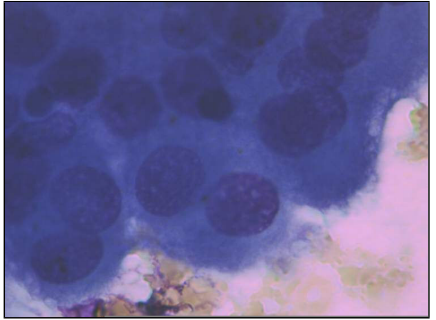

(a)

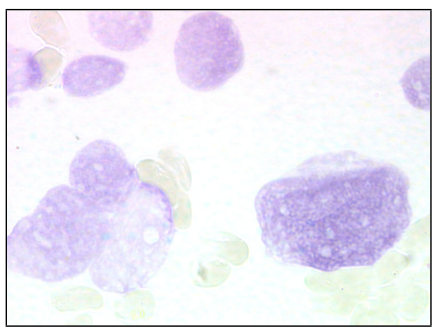

(d)

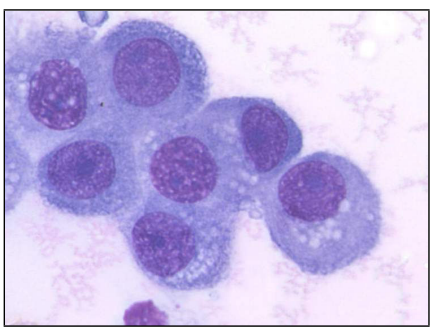

(g)

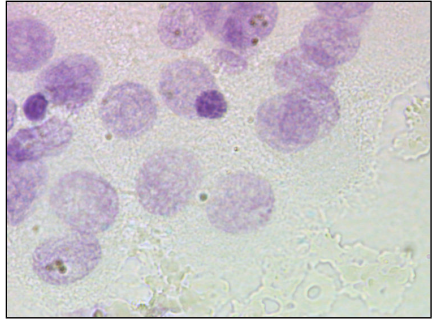

(b)

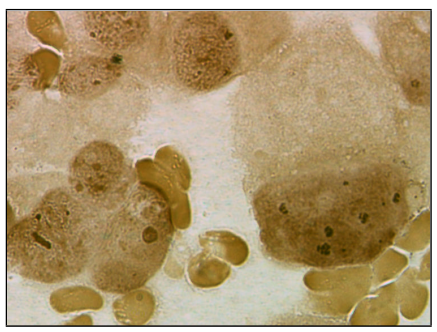

(e)

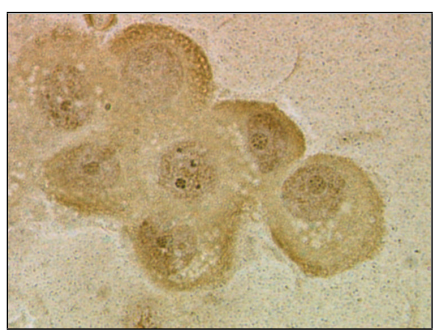

(h)

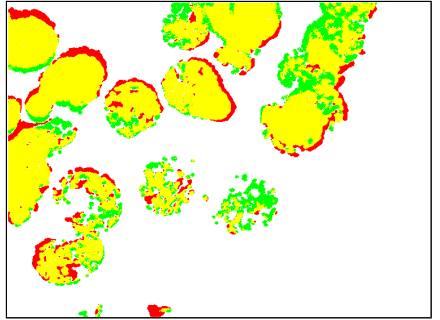

(c)

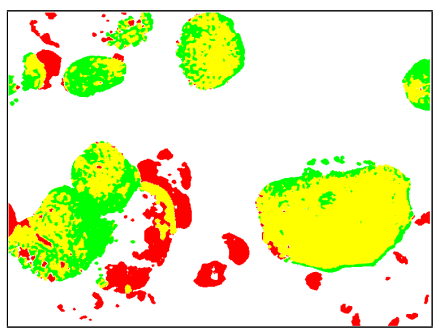

(f)

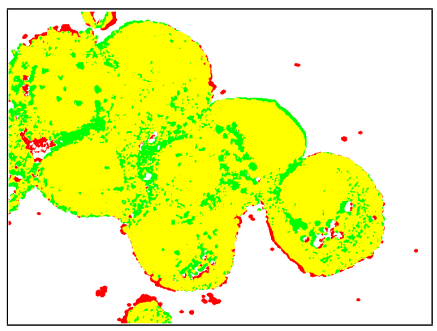

(i)

Fig. 2. Source images and overlaid mask images of successful scene comparisons: (a) MGG, (b) FEU, (d) FEU, (e) SIL, (g) MGG, (h) SIL, (c, f, i) Overlays, common foreground in yellow (resp. light grey), different foreground in green and red (resp. grey and dark grey)

pirically determined linear combinations of the colour channels. For MGG and SIL stains the background is masked out (see Fig. 1 (b)) using a threshold according to Otsu [3] (simplified as described in [4]), and a second application splits the nuclei from the plasma Fig. 1 (c). An example of a resulting binary image is shown in Fig. 1 (d). Since FEU does not stain the plasma a single threshold using the triangle algorithm [5] is sufficient.

A morphological closing (disc radius: 4px for MGG, 3px for FEU, 5px for SIL) followed by a flood filling is applied to close coves in the nuclei boundaries (see Fig. 1 (e)). For removal of small artefacts as well as long but thin filament-like objects without further morphological damage of the shape of the nuclei, a mask is generated using an opening (disc-radius: 15px). To include the nuclei boundaries a dilation (disc-radius: $5 \mathrm{px}$ ) of the mask has to follow (see Fig. 1 (f)). The mask is then AND-related with the prior binary image. The result is shown in Fig. $1(\mathrm{~g})$.

Finally shape criteria are used to separate overlapping and tangent nuclei. Single nuclei are convex while clusters mostly have concave spots. Therefore a distance transformation is used, which calculates the distance to the background for each pixel. This results in an image with dark spots at the center of each nucleus 1 (h) (inverted image). Applying the watershed transformation to this image separates clusters of nuclei to a satisfying degree. The background is masked out from the resulting image using $1(\mathrm{~g})$. Fig. 1 (i) shows the result in which two connected nuclei have been successfully separated.

\subsection{Scene Comparison and Matching}

The goal of the scene comparison is to determine a possible (partial) matching of the two scenes and thus to get the corresponding translation vector. This is achieved by matching the mask images of the nuclei. The best match of two scenes is found, whether or not the scenes are identical. The decision about identity of the two matched scenes is made by the succeeding image comparison. 
Matching the masks: Each nucleus of mask $g_{1}$ is superimposed on each one of mask $g_{2}$ using their centres of mass. This full search is not time-critical due to the low nuclei count in each image. For each pair the quality measure $Q_{1}$ is computed as follows:

$$
Q_{1}=\frac{A_{\text {small }}}{A_{\text {big }}} \cdot \sum_{x, y}\left(g_{1}(x, y) A N D g_{2}(x, y)\right)
$$

where $A_{\text {small }}$ is the area of the smaller nucleus and $A_{b i g}$ the area of the bigger one. The sum is a criterion for the total overlap of both masks, while the quotient $A_{\text {small }}$ over $A_{b i g}$ gives a local measure for the fit. The displacement vector is derived from the pair with the highest quality. If at least one nucleus in each stain is segmented with satisfying accuracy, the proper matching is found. All further operations are performed within the common image sections according to the match. A fine adjustment of the match is done by registering the greyscale images of the scenes using the mutual information criterion [6]. Since a translation vector is found even if the images contain different scenes the identity of the two scenes has to be tested as follows.

Image comparison: The identity test is based on a refinement of the pre-segmentation which exploits the correspondencies in different stains. The number of foreground pixels of the stain which on the average yields the superior pre-segmentation is used to get a better segmentation of the more problematic stain. This is achieved simply by varying the threshold of the latter towards reaching equal pixel counts in both stains. In the case of identity of the objects the segmented images are very similar, otherwise they appear different.

From the two binary images a further quality measure $Q_{2}$ is obtained as follows:

$$
Q_{2}=\frac{\sum_{x, y}\left(g_{1}(x, y) A N D g_{2}(x, y)\right)}{\sum_{x, y}\left(g_{1}(x, y) O R g_{2}(x, y)\right)}
$$

A perfect match will result in a value of 1. Examples for the image comparisons are shown in Fig. 2. The value of $Q_{2}$ is compared with an empirically acquired limit to decide whether the scenes are identical. If they are, the stage position is corrected according to the translation vector and the image is grabbed. Otherwise the surrounding area is sampled with a search strategy for the sought-after scene.

\section{RESULTS}

To relocate scenes with an accuracy of about $1 \mu \mathrm{m}$ an average of 1.6 iterations of scene comparison and correction of the position was necessary. Two of the eight slides showed unexplainable inconsistencies in the stored coordinates and could not be evaluated. The success rate of the other slides was $85.4 \%$ (1328/1556 scenes). This incorporates $7 \%$ of cases in which the search had to be extended to the surrounding area. $14.3 \%$ of the scenes could not be matched and no image was retrieved. $0.3 \%$ of the images were retrieved by mistake and showed different scenes. This occurs mostly in images which show only a single nucleus. Three examples of successful scene comparisons are shown in Fig. 2.

\section{SUMMARY AND DISCUSSION}

It could be shown that it is possible to automate the relocation process of re-stained cell samples which is required for multimodal cell analysis. The described scene comparison leads to satisfying results even if there are overlapping cells or if plasma is segmented by mistake. The $0.3 \%$ of wrongly matched scenes can be ascribed to images which show only a single nucleus in most cases. This failure rate can be further reduced by enlarging the FOV. Cause of the $14.3 \%$ unrelocated scenes is predominantly an insufficient pre-segmentation leading to inaccurate scene information. The success rate can potentially be improved by an enhanced pre-segmentation which makes better use of the local information of the images.

\section{REFERENCES}

[1] T. W. Remmerbach, H. Weidenbach, A. Hemprich, A. Böcking, "Earliest detection of oral cancer using non-invasive brush biopsy including DNA-imagecytometry: report on four cases.", Anal. Cell. Pathol.(25), 159-166, 2003

[2] A. Böcking, J. Stockhausen, D. Meyer-Ebrecht, "Towards a single cell cancer diagnosis. Multimodal and Monocellular Measurements of Markers and Morphology (5M)", Cell. Onc., 26, 73-79, 2004

[3] N. Otsu, "A threshold selection method from gray level histograms", IEEE TSMC(9), 62-66, 1979

[4] T. Hanning, "Vektorielle Mehrniveaupassung - Anwendungen in der Bildsegmentierung.", Ph.D. thesis, Universität Passau, Germany, 2002

[5] G. W. Zack, W. E. Rogers, S. A. Latt, "Automatic measurement of sister chromatid exchange frequency", J. Histochem. Cytochem.(25), 741-753, 1977

[6] P. Thévenaz, M. Unser, "A Pyramid Approach to SubPixel Image Fusion Based on Mutual Information", Proc. IEEE ICIP(I), 265-268, 1996 